\title{
Processing of poultry farm waste by microbial conversion
}

\author{
Irina Zanina ${ }^{1}$, Eugenia Kostromina ${ }^{1, *}$, Natalia Stuzhenko $^{1}$, and Yuri Chertov ${ }^{1}$ \\ ${ }^{1}$ Institute of service and business (branch) DSTU in Shakhty, Shevchenko str., 147, Shakhty, 346500, \\ Russia
}

\begin{abstract}
The article presents the results of an analysis of modern research in the field of organic waste processing. To solve the problem, anaerobic bioconversion method was chosen. The data of experimental laboratory experiments on the selection of a consortium of microorganisms' methanizing waste from poultry farms with maximum efficiency are presented.
\end{abstract}

\section{Introduction}

Currently, practically all ecosystems of the planet undergo greater or lesser degree of degradation under the influence of anthropogenic factors, in particular the accumulation of waste. The problem of waste accumulation and the search for promising methods of their processing is an urgent global problem [1].

The rapid development of poultry farming in recent years aimed at solving the problems of food security and import substitution has led to aggravation of the problem of organic waste accumulation. The impact on ecosystems in the zone of functioning of agricultural enterprises leads to ecological misbalance and their gradual degradation.

Large poultry farms negatively affect all components of the biosphere; their impact is comparable to large production facilities. About 773 million tons of agricultural wastes are generated annually in the Russian Federation, with 250 million tons of manure and 25 million tons of litter of them [2].

Organic waste includes feed residues, manure and litter, annual damage from agricultural waste is about 450 billion rubles [3].

The main environmental pollutant characteristic of poultry farms is litter.

It is recycled, as a rule, by removal to fields with subsequent plowing into the soil. The amount and properties of manure and litter depend on age, feeding diets and animal maintenance methods.

To preserve and maintain soil fertility, it is required to bring to the fields more than 15 tons of manure per 1 ha annually, which sometimes exceeds the cost of its transportation and application.

Initially birds' litter is also a source of pathogenic micro flora, containing weed seeds, and has a sharp unpleasant smell. Difficulties in using birds' litter in its initial form are also

*Corresponding author: kostromina_evgenia@mail.ru 
caused by its uneven distribution over the fertilized surface, as a result it leads to the death of plants due to a burn of the root system.

When used in livestock complexes and poultry farms without bedding, manure and litter are diluted 10-15 times with water, resulting in waste disposal and storage problems. It should be noted that liquid manure-containing effluents cause significant damage to the surface hydrosphere being a source of unpleasant smells.

Significant volumes of litter accumulated near poultry farms are practically not utilized, since poultry farms do not have available technologies and equipment for the beneficial use of such litter quantities. However, poultry litter is raw material to obtain high-quality organic fertilizers, biogas [4-7]. Thus, the most urgent task is the improvement and implementation of environmentally friendly methods of processing litter and manure [1].

In most EU countries, in accordance to environmental legislation, organic waste or even waste containing at least $5-10 \%$ of organic matter must be recycled. According to statistical studies, in the EU countries it can be concluded that the volume of waste, including organic origin, continues to increase steadily [10-13].

Switzerland, Austria, the Netherlands, Germany, Belgium and Norway are the leaders in utilizing and recycling of organic wastes.

There were practically no biotechnologies for waste processing in Russia until 2000. At the moment, the situation has changed; such technologies for using wastes as a raw material base for valuable products are being developed and improved [8-13].

Thus, the development of modern and highly efficient technologies for the disposal of poultry farm wastes should take into account such factors as:

-Compliance with veterinary and sanitary requirements to prevent infectious diseases in humans and animals;

-Production of valuable and environmentally safe products;

-Protection of the biosphere from pollution resulting from wastes processing.

All above mentioned requirements are satisfied by the method of processing manure and litter using biotechnologies - anaerobic biotransformation, which is a multistage process of splitting an organic substrate by a consortium of microorganisms with the formation of biogas under thermophilic or mesophilic conditions, which is an alternative source of energy and fermentation sludge that can be used as fertilizer.

The widespread introduction of technologies for producing biogas from agricultural waste through microbial conversion in Russia would make it possible to obtain up to 85 million tons of fuel per a year. However, the implementation of this program requires bioenergy installations with a total reactor capacity of 50-60 million $\mathrm{m}^{3}$.

In the EU countries (Finland, Sweden, Austria) the percentage of biogas energy from organic waste reaches $20 \%$. In Denmark, about $12 \%$ of the energy comes from organic waste. The Ministry of Energy of Russia plans to reach $10 \%$ of energy from alternative sources of all types only by 2030

The effectiveness of anaerobic fermentation of litter and manure can be enhanced by the addition of various ingredients to the original substrate (e.g. straws to chicken litter), careful grinding of raw materials, pre-treatment of waste by various methods. Hydrolysis, enzymatic, high temperature hydrolysis, treatment with acidic or alkaline agents can be used as pre-treatment methods, and the use of improved anaerobic reactor designs also contributes to the intensification of the process.

Anaerobic methanogenic consortium of mixed cultures of microorganisms is the most suitable for the destruction of organic matter of litter and manure of poultry farms. The biotechnological potential of consortia of microorganisms is constantly growing, finding the widest application in almost all sectors of the economy [8].

Biodegradation of organic substances is a multi-stage process in which various groups of microorganisms are involved. 
The quantitative and qualitative composition of the micro flora of the methanogenic community is determined by the composition of fermented organic substances and the process conditions. The process of methanization of organic substances (bird manure and litter) is carried out by a consortium of methanogenic bacteria and is divided into several trophically determined stages: enzymatic hydrolysis, acid formation, acetogenesis and methanogenesis (Figure 1).

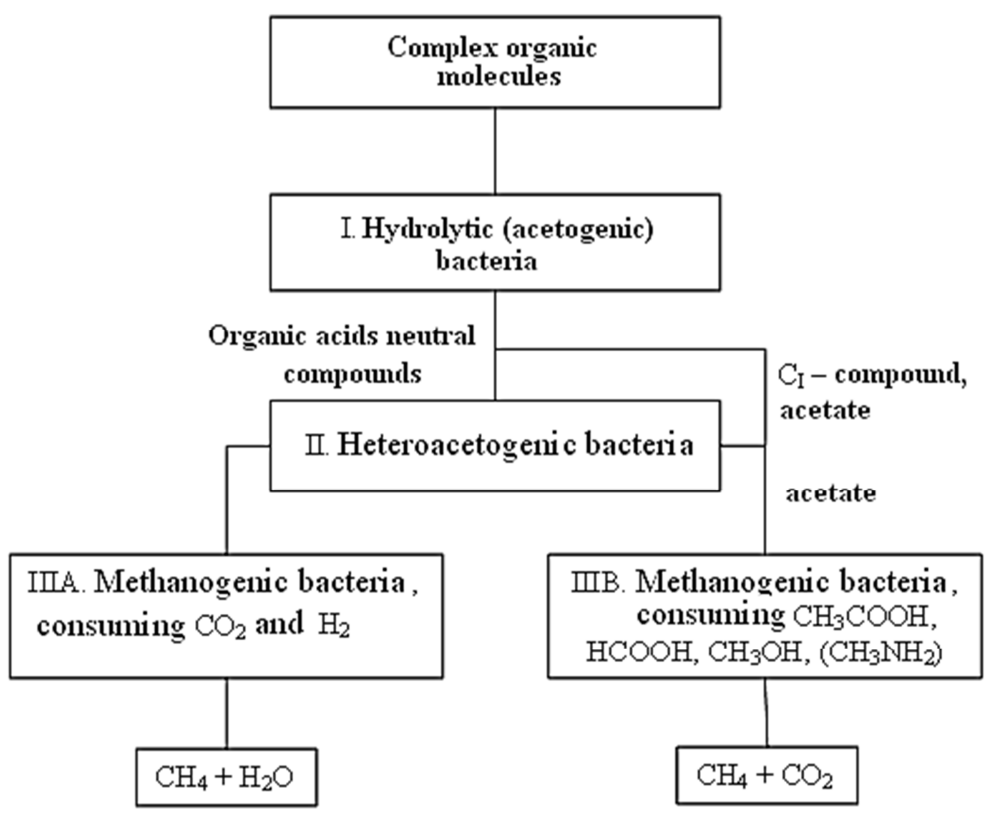

Fig. 1. Groups of bacteria responsible for various stages of biodegradation of organic substrates.

To ensure the optimal flow of the process, it is advisable to use the separation of the stages of acid formation and methane generation. The block diagram hab the form shown in Figure 2.

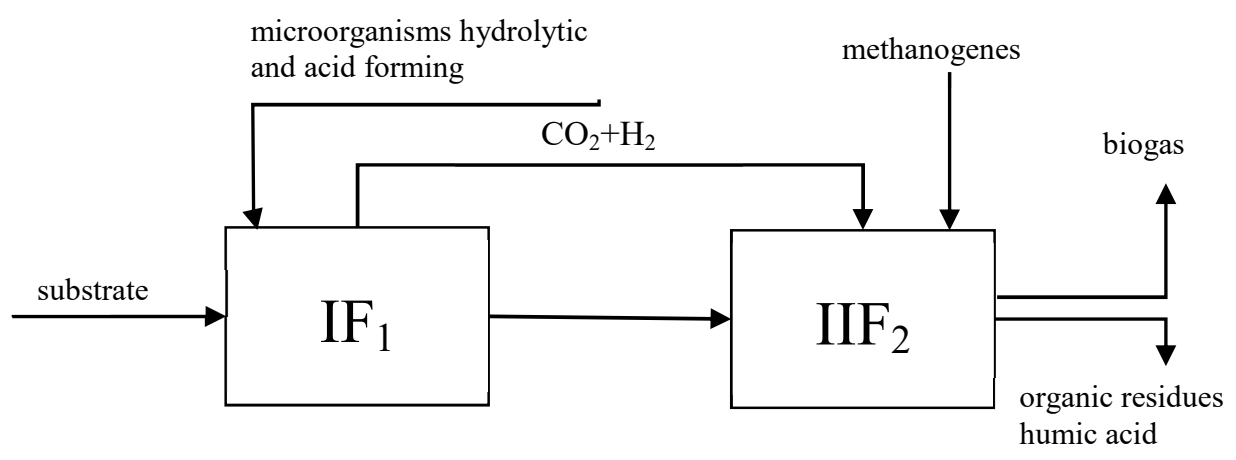

Fig. 2. Structural scheme of birds' litter conversion at separation of process stages.

The proposed structural scheme of bioconversion of poultry farm wastes with separate cultivation of fermentation and methanogenic microflora, will allow optimizing various process parameters: $\mathrm{pH}$, buffer capacity of medium, temperature regime, content of basic nutrients.

For laboratory studies, two microbial communities were selected to carry out microbial degradation of poultry farm wastes: I - a group of cultures is represented by a pair of $\mathrm{Cl}$. 
thermoccellum Methanobacterium thermoformiclum, organism $\mathrm{Cl}$. Thermococcellum decomposes complex organic substrates to form hydrogen, carbon dioxide, and ethanol. Methanogens from the genus Methanobacterium synthesize methane using mainly hydrogen and carbon dioxide as substrate.

Anaerobic community of microorganisms isolated from the sludge digester can be used for biotransformation of the waste. In terms of species diversity, the methane tank biocenosis contains about 50 species of bacteria responsible for the acid formation stage and a variety of methanogenic microflora. This ensures the success of biotransformation of the most complex organic substrates

Considering numerous successful experiments on using mold fungi in biotransformation processes, it is possible to use fungus culture at the pre-treatment stage. As. niger.

A series of experiments made it possible to obtain data determining the further direction of laboratory research. All methane release curves had a characteristic shape similar to curves during the destruction of other organic substrates. $n$ the first series of experiments (Figure 3), the best methane performance was achieved with methanization of chicken litter by the anaerobic consortium of methane tank $(58 \%$ methane on the 13 th day of cultivation).In the second place is methane yield during the conversion of turkey litter by a group of cultures $(\mathrm{Cl}$. Thermoccellum Methanobacterium thermoformiclum) $-52 \%$ on the 12th day of cultivation. The methanogenesis curves of duck litter showed a controversial result, the lowest methane yield was observed when using a group of cultures $(\mathrm{Cl}$. Thermoccellum Methanobacterium thermoformiclum) during biotransformation of duck litter by the methane tank consortium on the 10th day, methane yield reached a maximum of $51 \%$, and then there was a decrease in methane formation.

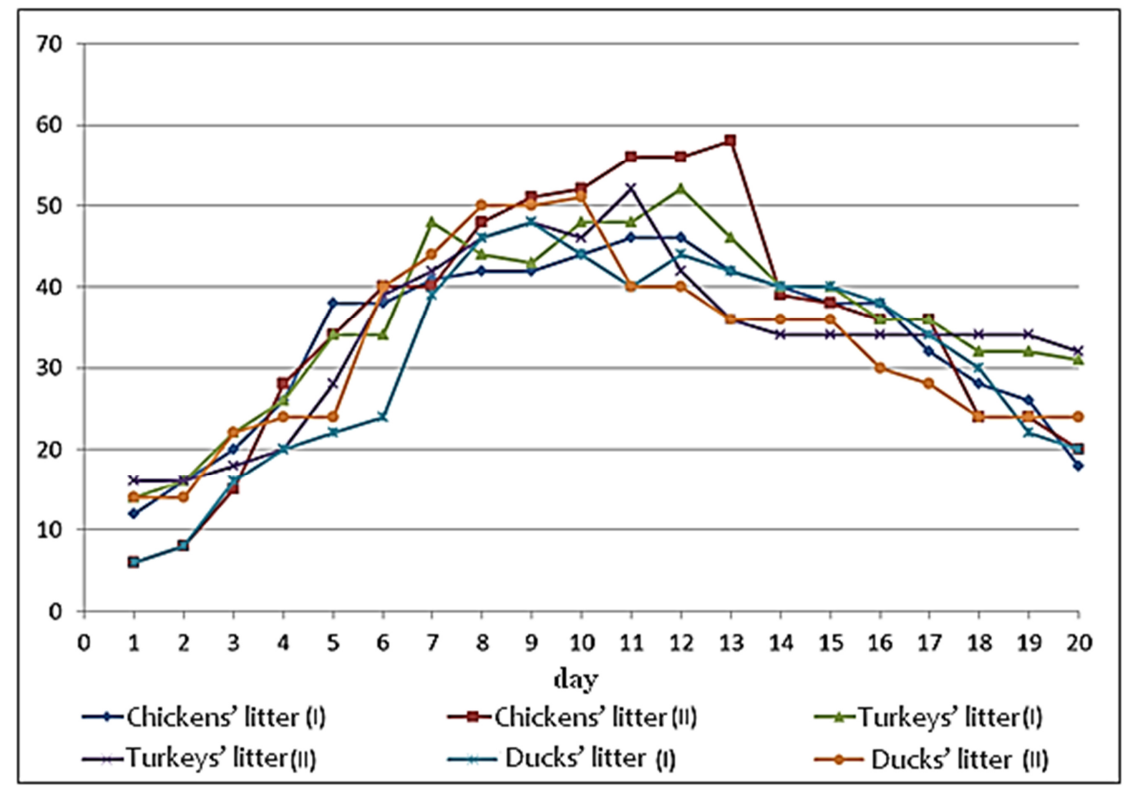

Fig. 3. Kinetics of birds' droppings methanogenesis.

The experience with the preliminary aerobic transformation of the chicken substrate by the fungal culture and further methanization by the anaerobic methane consortium allowed raising the methane yield to $62 \%$, accelerating the methanization process and achieving a stable process (Figure 4). 


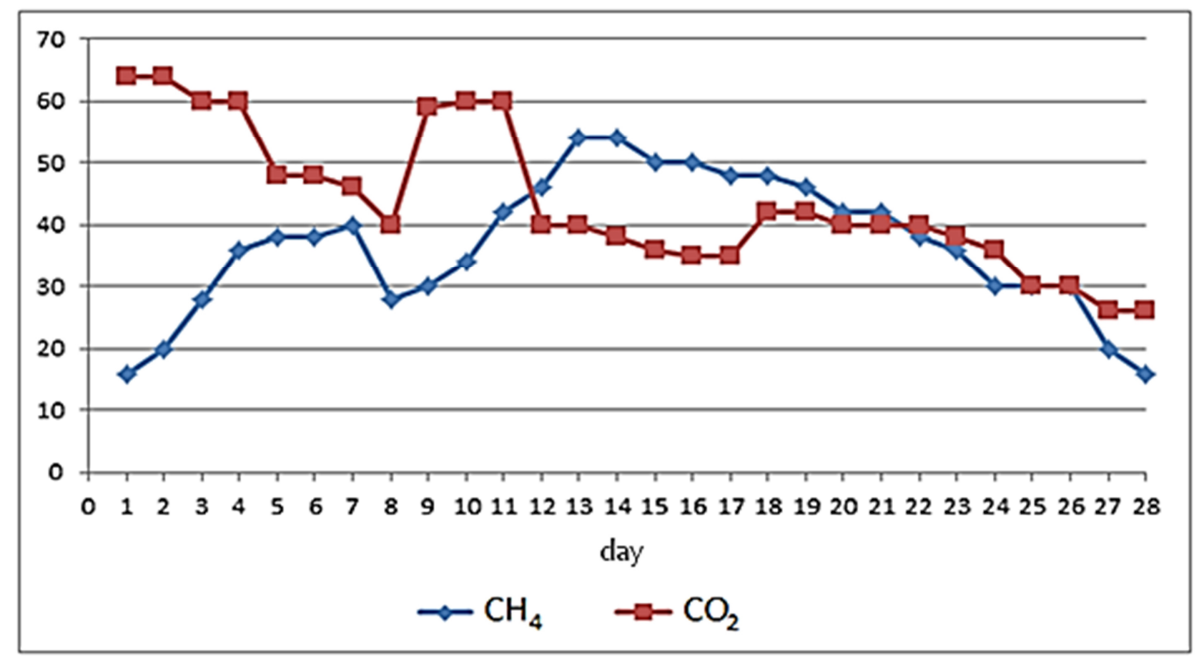

Fig. 4. Kinetics of gas release of chicken litter by the 1 group of crops $(\mathrm{Cl}$. thermoccellum Methanobacterium thermoformiclum).

It is obvious that preliminary bio-liquefaction by fungal culture contributes to the intensification of the methanization process. Biotechnological transformation is possible both by aerobic and anaerobic transformation. The use of fungal cultures, in particular the culture of the fungus As. niger followed by anaerobic transformation is shown in Figure 5.

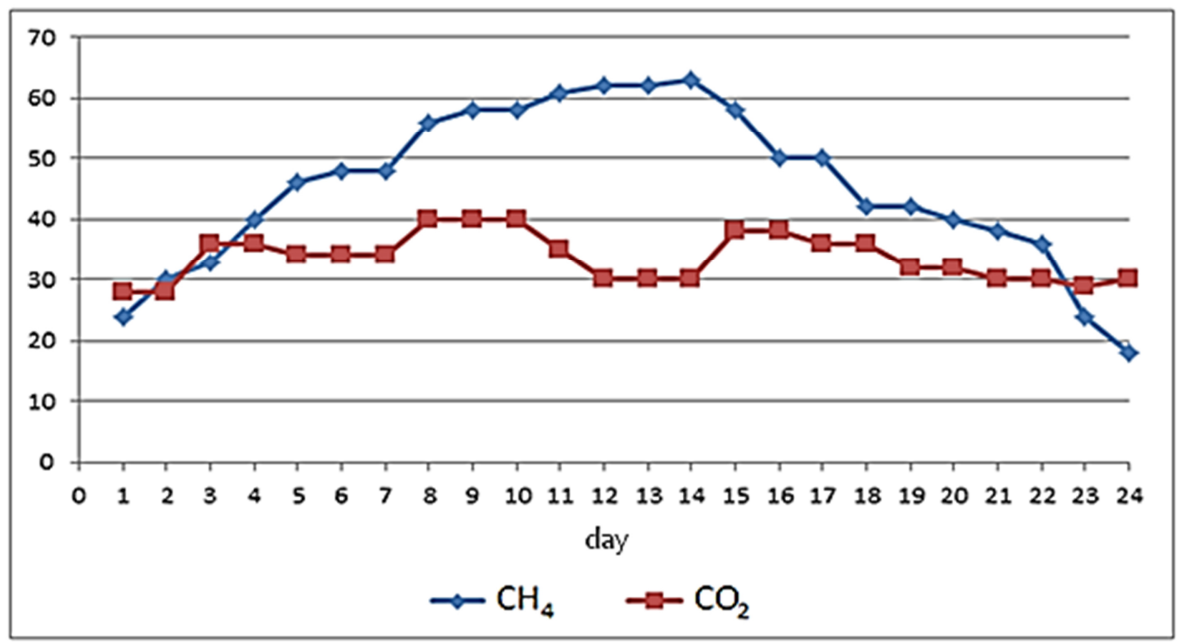

Fig. 5. Kinetics of methanogenesis of turkey litter with pre-treatment fungus culture As. Niger.

The positive effect of the separation of the acid and methane generation stages on the process of anaerobic digestion of waste was established.

A comparison of the results of experiments performed on the installation and without it showed an increase in methane yields for the methane tank consortium in the ratio 1: 1.05 $(5 \%)$ and for the group of cultures I - 1: 1.22. In addition, the separation of stages allowed increasing the duration of methane generation with sufficiently high methane content from 42 to $52 \%$ for 11 days.

$\mathrm{PH}$ control of the medium confirmed that the optimal $\mathrm{pH}$ for bioconversion of poultry waste was between 7.5 and 7.7. The introduction of a fresh portion of the inoculums on 
days 22-24 of the process leads to the appearance of a new peak in the curves of gas evolution.

As a result of the studies, the urgent task of improving the process of bioconversion of poultry wastes into biogas was solved by selecting and forming the biocenosis of the most active microorganisms, which will solve effectively the environmental problems of the accumulation of poultry waste and significantly improve the environmental situation in areas of poultry farms.

\section{References}

1. M.D. Molev, I.A. Zanina, A.G. Iliev, Y.E. Chertov, Astra-salvensis-supplement 1, 139150 (2017)

2. M. Molev, I. Zanina, Y. Chertov, A. Shemetov, MATEC Web of Conferences 170, 0419 (2018) doi: 10.1051/matecconf/201817004019

3. M.D. Molev, A.A. Kovaleva, I.A. Zanina, N.I. Stuzhenko, Yu.E. Chertov, Proceedings of the International Symposium "Engineering and Earth Sciences: Applied and Fundamental Research" dedicated to the 85th anniversary of H.I. Ibragimov (ISEES 2019) 1, 80-84 (2019) doi: 10.2991/isees-19.2019.17

4. J. Zabranska, D. Pokorna, Biotechnology Advances 36(3), 707-720 (2018) doi:10.1016/j.biotechadv.2017.12.003

5. B. Hua Yan, A. Selvam, J.W.C. Wong, Bioresource Technology 217, 3-9 (2016) doi: 10.1016/j.biortech.2016.03.116

6. E. Tsavkelova, L. Prokudina, M. Egorova, M. Leontieva, D. Malakhova, A. Netrusov, Process Biochemistry 66, 183-196 (2018) doi: 10.1016/j.procbio.2017.12.006

7. J. Fuertez, G. Córdoba, J.D. McLennan, D.J. Adams, T.D. Sparks, International Journal of Coal Geology 188, 165-180 (2018) doi: 10.1016/j.coal.2018.02.013

8. J. Fuertez, R. Boakye, J. McLennan, D.J. Adams, T.D. Sparks, A. Gottschalk, Journal of Natural Gas Science and Engineering 46, 637-650 (2017) doi: 10.1016/j.jngse.2017.07.028

9. L.A. Soares, C.A.B.S. Rabelo, T.P. Delforno, E.L. Silva, M.B.A. Varesche, Renewable Energy 140, 852-861 (2019) doi: 10.1016/j.renene.2019.03.103.

10. S. Mirmohamadsadeghi, K. Karimi, M. Tabatabaei, M. Aghbashlo, Bioresource Technology Reports 7 (2019) doi: 10.1016/j.biteb.2019.100202

11. L. Zhang, K.-C. Loh, J. Zhang, Bioresource Technology Reports 5, 280-296 (2019) doi: 10.1016/j.biteb.2018.07.005.

12. Y. Xu, Y. Lu, L. Zheng, Z. Wang, X. Dai, Journal of Hazardous Materials (2019) doi: 10.1016/j.jhazmat.2019.121847

13. J.-K. Lee, S. Kumar Singh Patel, B. Hyun Sung, V.C. Kalia, Bioresource Technology 298 (2020) doi: 10.1016/j.biortech.2019.122346 